\title{
Polymerization of Propiolaldehyde. II. Radical Copolymerization of Propiolaldehyde with Styrene
}

\author{
Kazukiyo КовауAShi and Hiroshi Sumitomo \\ Faculty of Agriculture, Nagoya University, Chikusa, Nagoya 464, Japan. \\ (Received March 17, 1972)
}

\begin{abstract}
Radical copolymerization of propiolaldehyde $\left(\mathrm{M}_{B}\right)$ with styrene $\left(\mathrm{M}_{\mathrm{A}}\right)$ was carried out at $60^{\circ} \mathrm{C}$ over a wide range of monomer feed compositions. It was shown from the results of IR, NMR, and UV spectral analyses of the copolymers that propiolaldehyde was copolymerized through the acetylenic opening to give the conjugated enal unit, $-\mathrm{CH}=\mathrm{C}-$, and that the copolymerization had a rather high
\end{abstract}

tendency to alternation. The behavior is also in agreement with the results predicted by the statistically calculated parameters such as dyad fraction, average sequence length, and run number. Monomer reactivity ratios $\left(r_{\mathrm{A}}=0.32\right.$ and $\left.r_{\mathrm{B}}=0.21\right)$ and the $Q$ and $e$ values $(Q=0.83$ and $e=0.84)$ are compared with those of some acetylenic monomers and the corresponding vinyl monomers. Propiolaldehyde is found to show the highest polymerization reactivity among the acetylenic monomers. This may be due to the activation of the acetylenic group by conjugation with the electron-withdrawing aldehyde group.

KEY WORDS Radical Copolymerization / Propiolaldehyde Styrene / Acetylenic Opening / Monomer Reactivity Ratios / Alternating Tendency /

It was reported in the previous paper, ${ }^{1}$ that propiolaldehyde, the simplest ynal $\mathrm{CH} \equiv \mathrm{C}-\mathrm{CH}=\mathrm{O}$, was polymerized through the aldehyde opening in the presence of sodium cyanide at $-78^{\circ} \mathrm{C}$ to give the crystalline poly[(ethynyl)oxymethylene]. The unique reactivity of the aldehyde and the acetylene group toward a nucleophile was noted. On the other hand, radical polymerization of the monomer was also described briefly.

Acetylenic monomers are generally not as reactive as vinyl monomers and produce lower molecular weight polymers. Nevertheless, attempts have been made to prepare a variety of polymers having the conjugated polyene structure from acetylenic compounds for the purpose of the utilization of their possible semiconductivity. Coordination-type catalysts such as Ziegler-Natta-type complexes and transition metal complexes were found to be effective for the polymerization of acetylenic monomers, but little was reported on their radical polymerization. As to the copolymerization with vinyl monomers, information has been so far provided regarding only phenylacetylene,$^{2-5}$ diphenylacetylene, ${ }^{2} n$-hexyne-1, ${ }^{2}$ and propargyl alcohol. ${ }^{5}$ Furthermore, it should be noted that a copolymer could be obtained only in the case of a relatively low feed-ratio of acetylenic monomer.

In the present work, it was found that the copolymerization of propiolaldehyde with styrene can be performed over a wide range of monomer feed compositions. The acetylenic group of propiolaldehyde is clearly activated by conjugation with the aldehyde. Estimations of copolymerization parameters, analyses of microstructure of the resulting copolymers and comparison of copolymerization reactivity between acetylenic and vinyl monomers are reported and discussed.

\section{EXPERIMENTAL}

\section{Materials}

Propiolaldehyde was prepared by oxidation of propargyl alcohol with chromic acid., ${ }^{1,6}$ The aldehyde was dried over anhydrous magnesium 
sulfate and was purified by fractional distillation in a stream of nitrogen gas and then by vacuum distillation just before use.

Styrene was washed three times with potassium hydroxide and with water, and then dried over anhydrous potassium carbonate and distilled just before use.

Azobisisobutyronitrile (AIBN) was recrystallized from ethanol and dried in vacuo.

\section{Copolymerization}

The mixture of propiolaldehyde and styrene (total amount of monomers, $0.05 \mathrm{~mol}$ ) was put into the glass ampule containing $41 \mathrm{mg}$ of AIBN ( $0.5 \mathrm{~mol} \%$ to monomers). After being degassed three times and sealed, the ampule was allowed to stand in a thermostat kept at $60 \pm 0.02^{\circ} \mathrm{C}$. The conversion was controlled to lower than $10 \%$. Methanol or ether was used as a precipitating reagent. The copolymer was purified by reprecipitating from tetrahydrofuran (THF)methanol or-ether.

\section{Characterization of Copolymers}

The mole fraction of propiolaldehyde unit in copolymer was calculated from the carbon content as determined by the elemental analysis. The number-average molecular weight of the copolymer was determined by vapor pressure osmometry in benzene or $N, N$-dimethylformamide (DMF) at $37^{\circ} \mathrm{C}$ using a Hewlett-Packard Model 302. Infrared spectra were measured as $\mathrm{KBr}$ disk with a Hitachi grating infrared spectrophotometer model 215. NMR spectra were recorded at $70^{\circ} \mathrm{C}$ with a Japan Electron Optics Lab. Model JNM-4H-100 high resolution spectrometer at $100 \mathrm{MHz}$. Tetramethylsilane was used as an internal reference for spectra obtained for deuteriochloroform $(5 \%)$ or deuterioDMF (10\%) solution. Electronic spectra were recorded with a Shimadzu multipurpose recording spectrophotometer MPS-50. Differential thermal analyses of the copolymers were carried out on a Rigaku-Denki 8001 DTA apparatus.

\section{RESULTS AND DISCUSSION}

\section{Copolymerization}

Table I summarizes the results of radicalinitiated copolymerization of propiolaldehyde $\left(M_{B}\right)$ with styrene $\left(M_{A}\right)$. Both the polymerization rate and the molecular weight of the resulting polymer were lowered with increasing mole fraction of propiolaldehyde in the feed composition. The copolymer obtained is a powdery solid that is soluble in DMF and THF. The copolymer with a lower content of propiol-

Table I. Radical copolymerization of propiolaldehyde $\left(\mathbf{M}_{B}\right)$ with Styrene $\left(\mathbf{M}_{\mathbf{A}}\right)^{\mathrm{a}}$

\begin{tabular}{ccccccccc}
\hline No. & $\begin{array}{c}\mathrm{M}_{\mathrm{A}}, \\
\mathrm{g}\end{array}$ & $\begin{array}{c}\mathrm{M}_{\mathrm{B}}, \\
\mathrm{g}\end{array}$ & $\begin{array}{c}\text { Mole fraction } \\
\text { of } \mathrm{M}_{\mathrm{B}}\end{array}$ & $\begin{array}{c}\text { Time, } \\
\mathrm{hr}\end{array}$ & $\begin{array}{c}\text { Conversion, } \\
\%\end{array}$ & $M_{n}$ & $\begin{array}{c}\text { Mole fraction } \\
\text { of } \mathrm{M}_{\mathrm{B}} \\
\text { in copolymer }\end{array}$ & $\varepsilon_{340}$ \\
\hline 48 & 5.21 & 0 & 0 & 4 & $25.1^{\mathrm{b}}$ & $12900^{\mathrm{d}}$ & - & - \\
49 & 4.70 & 0.289 & 0.106 & 11 & $7.5^{\mathrm{b}}$ & $5110^{\mathrm{d}}$ & 0.21 & 260 \\
53 & 4.17 & 0.564 & 0.207 & 15 & $7.0^{\mathrm{b}}$ & - & 0.32 & - \\
50 & 3.65 & 0.810 & 0.300 & 16 & $5.1^{\mathrm{b}}$ & $4250^{\mathrm{a}}$ & 0.38 & 510 \\
54 & 3.13 & 1.09 & 0.401 & 20 & $6.3^{\mathrm{b}}$ & - & 0.45 & - \\
51 & 2.60 & 1.44 & 0.516 & $\prime \prime$ & $5.8^{\mathrm{b}}$ & $2600^{\mathrm{e}}$ & 0.47 & 640 \\
71 & 2.02 & 1.63 & 0.609 & 27 & $9.3^{\mathrm{c}}$ & $1900^{\circ}$ & 0.55 & 870 \\
85 & 1.57 & 1.90 & 0.700 & 19 & $8.1^{\mathrm{c}}$ & $1630^{\circ}$ & 0.60 & 1000 \\
86 & 1.05 & 2.17 & 0.798 & $\prime \prime$ & $8.6^{\mathrm{c}}$ & $1270^{\circ}$ & $(0.79)^{\mathrm{f}}$ & - \\
87 & 0.534 & 2.45 & 0.898 & $\prime \prime$ & $7.8^{\mathrm{c}}$ & $1100^{\circ}$ & $(0.90)^{\mathrm{f}}$ & - \\
88 & 0 & 2.64 & 1.0 & $\prime \prime$ & $1.9^{\mathrm{c}}$ & $910^{\circ}$ & - & - \\
\hline
\end{tabular}

a Total amount of the monomers, $0.05 \mathrm{~mol}$; AIBN, $41 \mathrm{mg}(0.5 \mathrm{~mol} \%$ to monomers $), 60^{\circ} \mathrm{C}$.

b Precipitated with methanol.

- Precipitated with ether.

d VPO, benzene, $37^{\circ} \mathrm{C}$.

- VPO, DMF, $37^{\circ} \mathrm{C}$.

${ }^{f}$ Designated in parentheses owing to the poor reproducibility of the elemental analysis. 


\section{K. Kobayashi and H. Sumitomo}

aldehyde unit is also soluble in ether, benzene, and chloroform but insoluble in methanol. As the content of propiolaldehyde unit increased, the powdery polymer became colored in redbrown and its solubility in ether, benzene, and chloroform decreased. High propiolaldehyde-

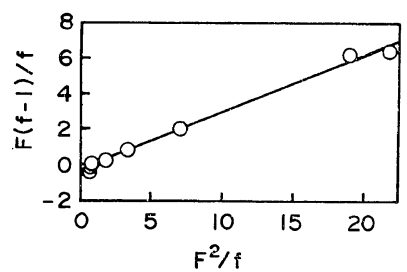

Figure 1. The Fineman-Ross plots for the copolymerization of propiolaldehyde $\left(\mathrm{M}_{\mathrm{B}}\right)$ with styrene $\left(\mathrm{M}_{\mathrm{A}}\right)$.

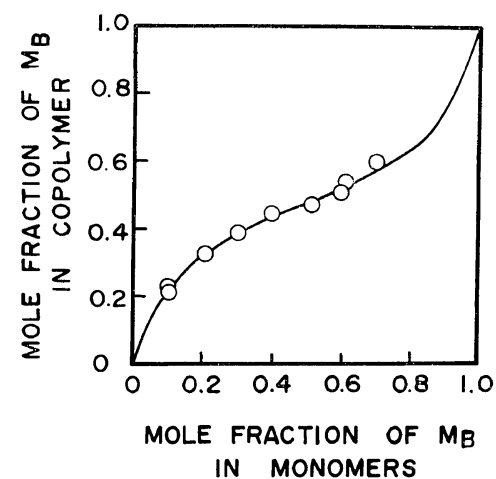

Figure 2. Composition curve for the copolymerization of propiolaldehyde $\left(\mathrm{M}_{\mathrm{B}}\right)$ with styrene $\left(\mathrm{M}_{\mathrm{A}}\right)$; AIBN, bulk, $60^{\circ} \mathrm{C}$.

$O$, experimental; - , calculated from $r_{\mathrm{A}}=0.32$ and $r_{B}=0.21$.

Table II. Statistically estimated copolymerization parameters

\begin{tabular}{ccccccc}
\hline No. & $\boldsymbol{P}_{2}\{\mathrm{AA}\}$ & $\begin{array}{c}\boldsymbol{P}_{2}\{\mathrm{AB}\} \\
=\boldsymbol{P}_{2}\{\mathrm{BA}\}\end{array}$ & $\boldsymbol{P}_{2}\{\mathrm{BB}\}$ & $\overline{1}_{\mathrm{A}}$ & $\overline{1}_{\mathrm{B}}$ & $R$ \\
\hline 49 & 0.57 & 0.21 & 0.01 & 3.7 & 1.0 & 43 \\
53 & 0.37 & 0.31 & 0.02 & 2.2 & 1.1 & 61 \\
50 & 0.26 & 0.35 & 0.03 & 1.7 & 1.1 & 71 \\
54 & $\mathbf{0 . 1 8}$ & 0.38 & 0.06 & 1.5 & 1.1 & 76 \\
51 & 0.12 & 0.40 & 0.09 & 1.3 & 1.2 & 79 \\
71 & 0.08 & 0.39 & 0.13 & 1.2 & 1.3 & 79 \\
85 & $\mathbf{0 . 0 5}$ & 0.38 & 0.19 & 1.1 & 1.5 & 76 \\
86 & 0.03 & 0.34 & 0.29 & 1.1 & 1.9 & 68 \\
87 & 0.01 & 0.25 & 0.48 & 1.0 & 2.9 & 51 \\
\hline
\end{tabular}

content copolymer is soluble in methanol and acetone.

The Finemann-Ross plots gave a straight line as shown in Figure 1. The monomer reactivity ratios were estimated to be $r_{\mathrm{A}}=0.32$ and $r_{B}=0.21$. Figure 2 illustrates that the calculated composition curve for the copolymer agrees with the experimental plots. Assuming that the copolymerization follows the statistical steady state, dyad fractions $\left(P_{2}\{\mathrm{AA}\}, P_{2}\{\mathrm{AB}\}\right.$, $\boldsymbol{P}_{2}\{\mathrm{BA}\}$, and $\left.\boldsymbol{P}_{2}\{\mathrm{BB}\}\right)$, average sequence length $\left(\bar{I}_{\mathrm{A}}, \bar{I}_{\mathrm{B}}\right)$, and run number $(R)$ were calculated from the molar feed-ratio and the reactivity ratios $\left(r_{\mathrm{A}}\right.$ and $\left.r_{\mathrm{B}}\right)$ according to the method of Ito and Yamashita ${ }^{7}$ (Table II). These values, the large $P_{2}\{\mathrm{AB}\}=P_{2}\{\mathrm{BA}\}, \bar{I}_{\mathrm{A}}$ and $\bar{l}_{\mathrm{B}}$ close to unity, and the large $R$, mean that the copolymerization has a tendency to alternate. The trend is evidenced by the analyses of the microstructure of the copolymers as described in the later section. The $Q$ and $e$ values of the Alfrey-Price equation were calculated to be $Q=0.83$ and $e=0.84$.

Characterization of Copolymers and Their Microstructure

The infrared spectra of propiolaldehyde and the copolymer are presented in Figure 3 (the propiolaldehyde content $\left(m_{\mathrm{B}}\right)$ is 0.47$)$. The absorptions at 3280,2100 , and $660 \mathrm{~cm}^{-1}$ assignable respectively to $\mathrm{C}-\mathrm{H}$ and $\mathrm{C} \equiv \mathrm{C}$ stretching and $\mathrm{C}-\mathrm{H}$ wagging vibration of acetylenic group were absent in the copolymer. The new peak appears at $1620 \mathrm{~cm}^{-1}$ assignable to a carboncarbon double bond conjugated to the aldehyde.

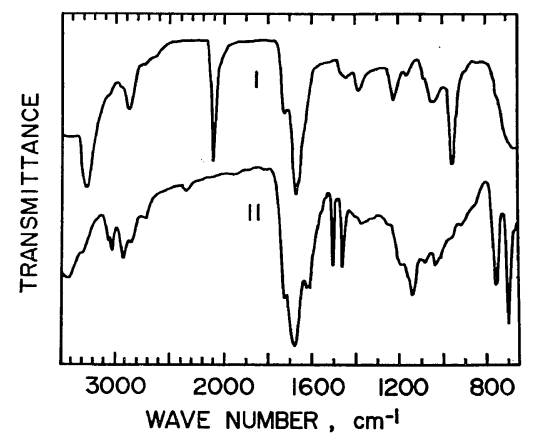

Figure 3. Infrared spectra of propiolaldehyde (I) (liquid film) and the copolymer (II) $\left(m_{\mathrm{B}}=\mathbf{0 . 4 7 )}\right.$ ( $\mathrm{KBr}$ disk). 
The peaks at $3060-2930,1600,1500,1455,755$, and $695 \mathrm{~cm}^{-1}$ show the presence of a styrene unit. It is suggested that propiolaldehyde is copolymerized with its acetylene group with styrene to give the enal unit.

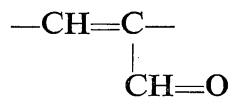

It was reported in the previous paper ${ }^{1}$ that the AIBN-initiated homopolymer of propiolaldehyde caused a slow reaction of the adjacent pendant aldehyde groups. The statistical data for the present copolymerization suggest that the content of the adjacent pendant aldehyde units in the copolymer is rather small. Therefore little reaction between aldehyde groups is assumed to occur. However, in the region of $m_{\mathrm{B}}$ higher than about 0.8 , the reproducibility of the elemental analysis of the copolymer became poorer. A shift of the conjugated double bond absorption to $1610 \mathrm{~cm}^{-1}$ and appearance of an acetal absorption at 1130 and $1190 \mathrm{~cm}^{-1}$ were also observed in their infrared spectra. The increase of the content of adjacent pendant aldehyde groups is assumed to lead to the formation of a dihydropyran ring structure.

It was found by differential thermal analysis that the copolymer (sample no. 49) exhibited an exothermic peak in the range of $125-165^{\circ} \mathrm{C}$. No weight loss was observed and the solubility and the infrared spectrum of the cooled product were almost the same as those of the original polymer. However, broad exothermic bands were observed in the range of $145-230^{\circ} \mathrm{C}$ for the copolymer (sample no. 50) and of 145$280^{\circ} \mathrm{C}$ for the one (sample no. 51). The cooled samples became insoluble in common organic solvents and exhibited a remarkable decrease in the intensity of the carbonyl absorption in the infrared spectra. Some complicated polymeric reactions such as cross-linking might be induced by heating.

It was observed in the NMR spectra that, although the phenyl proton signals were separated into two peaks at $\tau 3.00$ and 3.45 in homopolystyrene, the two peaks were combined together and shifted to a little lower field as the propiolaldehyde content increased (Figure 4 and Table III). When the fraction $P_{5}$ \{AAAAA calculated statistically was 0.02 (sample no. 50), the separation of the peaks almost disappeared. The result seems to agree approximately with the previously described assumption that separation of the phenyl proton signal occurs with five or more styrene units. A rather high tendency to alternate in the present system is suggested here too.

The electronic spectrum of the copolymer having a low content of propiolaldehyde exibits a weak shoulder around the visible region:

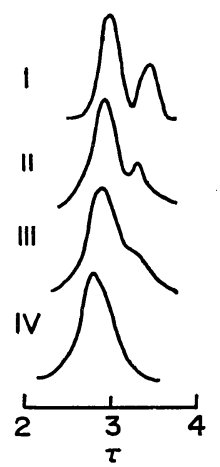

Figure 4. NMR spectra of polystyrene and propiolaldehyde-styrene copolymers: I, polystyrene, $60 \mathrm{MHz}, \mathrm{CCl}_{4}, 15 \%, 99^{\circ} \mathrm{C}$; II, III, and IV, copolymer, $100 \mathrm{MHz}, \mathrm{CDCl}_{3}, 5 \%, 70^{\circ} \mathrm{C}$ (for samples, see Table III).

Table III. NMR data of propiolaldehyde-styrene copolymers

\begin{tabular}{cccccccc}
\hline Spectrum & $\begin{array}{c}\text { Sample } \\
\text { no. }\end{array}$ & $\begin{array}{c}\text { Mole fraction } \\
\text { of } \mathbf{M}_{\mathrm{A}} \text { in } \\
\text { copolymer, } \\
m_{\mathrm{A}}\end{array}$ & $\begin{array}{c}\text { Mole fraction } \\
\text { of } \mathbf{M}_{\mathrm{B}} \text { in } \\
\text { copolymer, } \\
m_{\mathrm{B}}\end{array}$ & $\begin{array}{c}\text { Average chain } \\
\text { length of } \\
\mathbf{M}_{\mathrm{A}},\end{array}$ & $P_{5}$ \{AAAAA\} & $\begin{array}{c}\text { NMR shift of } \\
\text { phenyl group, } \\
\tau\end{array}$ \\
\hline I & - & 1.0 & 0 & - & 1.0 & 3.00, & 3.45 \\
II & 49 & 0.79 & 0.21 & 3.7 & 0.22 & 2.90, & 3.30 \\
III & 50 & 0.62 & 0.38 & 1.7 & 0.02 & 2.87, & Shoulder \\
IV & 51 & 0.53 & 0.47 & 1.5 & 0.001 & 2.80, & - \\
\hline
\end{tabular}


with sample no. 49 at $386 \mathrm{~m} \mu(\varepsilon, 140)$, sample no. 50 at $395 \mathrm{~m} \mu(\varepsilon, 290)$, and sample no. 51 at $400 \mathrm{~m} \mu(\varepsilon, 260)$. These absorptions would arise from the succession of the propiolaldehyde unit. However, with the copolymers of $m_{\mathrm{B}}>0.5$, it is masked by the strong absorption in the ultraviolet region. For convenience the molar absorptivities at $340 \mathrm{~m} \mu\left(\varepsilon_{340}\right)$ corresponding to one propiolaldehyde unit were calculated and listed in the last column of Table I. The molar absorptivity at $340 \mathrm{~m} \mu$ of $\mathrm{R}(\mathrm{CH}=\mathrm{CH})_{n} \mathrm{CH}=\mathrm{O},{ }^{10,11}$ although not so good as model compounds, are available: $n=0, \varepsilon \sim 0 ; n=1, \varepsilon \sim 30 ; n=2, \varepsilon \sim 800$; $n=3, \varepsilon \sim 4000$ (these values are estimated from the spectra shown in the literature). It is presumed that the structures of $n=1$ and 3 approximately correspond here to $\bar{I}_{\mathrm{B}}=1$ and 2 , respectively. The estimated $\varepsilon_{340}$ values of the present copolymers suggest that the content of a continuous B-unit sequence is rather small.

It is concluded from the spectral analysis that the present copolymerization of propiolaldehyde is performed through the acetylenic opening with a rather high tendency to alternate. The extent of agreement between the spectral data and the statistical aspects is also noticeable.

\section{Copolymerization reactivity}

Acetylenic monomers are generally much less reactive than the corresponding vinyl monomers. In addition, both acetylenic and aldehyde compounds are known to be chain-transfer reagents for radical polymerization. In fact, the increase of the mole fraction of propiolaldehyde in the feed decreased the polymerization rate and the molecular weight of the resulting polymers as shown in Table I. However, that the copolymerization of propiolaldehyde was found to proceed over the whole feed compositions had not reported so far for any other acetylenic monomers.

The parameters of radical copolymerization of propiolaldehyde, acrolein, ${ }^{12}$ and $\alpha$-cyanomethyl acrolein ${ }^{13}$ with styrene are compared in Table IV. The copolymerizations of acrolein and $\alpha$-cyanomethyl acrolein were carried out with the use of AIBN in dioxane at $50^{\circ} \mathrm{C}$ and of benzoyl peroxide in THF at $65^{\circ} \mathrm{C}$, respectively. The term $r_{A} r_{B}$ of propiolaldehyde is slightly smaller than that of acrolein, while not so small as that of $\alpha$-cyanomethyl acrolein. The term $1 / r_{A}$, which means the reactivity of $\mathrm{B}$ monomer to the styryl radical, follows the order: propiol-

Table IV. Comparison of copolymerization parameters

\begin{tabular}{clccccccc}
\hline $\mathrm{M}_{\mathrm{A}}$ & \multicolumn{1}{c}{$\mathrm{M}_{\mathrm{B}}$} & $r_{\mathrm{A}}$ & $r_{\mathrm{B}}$ & $r_{\mathrm{A}} r_{\mathrm{B}}$ & $1 / r_{\mathrm{A}}$ & $Q_{\mathrm{B}}$ & $e_{\mathrm{B}}$ & Ref \\
\hline Styrene & Propiolaldehyde & 0.32 & 0.21 & 0.067 & 3.12 & 0.83 & 0.84 & This paper \\
$\prime \prime$ & Acrolein & 0.22 & 0.33 & 0.073 & 4.54 & 1.25 & 0.82 & 12 \\
$\prime \prime$ & $\alpha$-Cyanomethyl acrolein & 0.11 & 0.06 & 0.0066 & 9.09 & 1.35 & 1.44 & 13 \\
\hline
\end{tabular}

Table V. $Q-e$ Values of acetylenic monomers and vinyl monomers

\begin{tabular}{|c|c|c|c|c|c|c|c|}
\hline Acetylenic monomer & $Q$ & $e$ & Ref & Vinyl monomer & $Q$ & $e$ & Ref \\
\hline $\mathrm{CH} \equiv \mathrm{C}-\mathrm{CH}=\mathrm{O}$ & 0.83 & 0.84 & This paper & $\mathrm{CH}_{2}=\mathrm{CH}-\mathrm{CH}=\mathrm{O}$ & 1.25 & 0.82 & 12 \\
\hline $\mathrm{c}$ & 0.35 & -0.66 & 14 & $\mathrm{CH}$ & 1.00 & -0.80 & - \\
\hline & 0.0025 & -1.23 & 14 & & 0.017 & -0.03 & 14 \\
\hline & & & & & 0.030 & -0.08 & 14 \\
\hline $\mathrm{CH} \equiv \mathrm{C}-\left(\mathrm{CH}_{2}\right)_{3} \mathrm{CH}_{3}$ & 0.014 & -0.70 & 14 & $\mathrm{CH}_{2}=\mathrm{CH}-($ & 0.019 & -0.28 & 14 \\
\hline
\end{tabular}


aldehyde $<$ acrolein $<\alpha$-cyanomethyl acrolein.

Acetylenic monomers seem to have the $Q$ values smaller than vinyl monomers, as compared in Table V. The highest $Q$ value of propiolaldehyde among the acetylenic monomers listed indicates the highest polymerizability. It is suggested that the conjugation with the aldehyde activates the acetylenic group for radical copolymerization.

It was reported that copolymerization with vinyl chloride by AIBN in THF at $70^{\circ} \mathrm{C}$ gave only a viscous liquid product. ${ }^{5}$ As compared with the system for vinyl chloride, for which the value is $0.20,{ }^{14}$ propiolaldehyde $(e=0.84)$ and styrene $(e=-0.80)$ of the present system exibit the larger difference in $e$ value. It may be concluded that the electrostatic attraction between the two monomers, coupled with the high polymerizability, gives rise to the alternating copolymerization of propiolaldehyde with styrene over the wide range of monomer feed compositions.

\section{REFERENCES}

1. K. Kobayashi and H. Sumitomo, J. Polym. Sci., Part B, 10, 703 (1972).

2. K. W. Doak, J. Amer. Chem. Soc., 72, 4681
(1950).

3. C. C. Price and C. E. Greene, J. Polym. Sci., 6, 111 (1951).

4. H. Higashiura, Kogyo Kagaku Zasshi (J. Chem. Soc. Japan, Chem. Ind. Sect.), 69, 1243 (1966).

5. S. Takahashi, K. Takemoto, and M. Imoto, Kobunshi Kagaku (Chem. High Polymers), 26, 374 (1969).

6. J. C. Sauer, Org. Syn., 36, 66 (1956).

7. K. Ito and Y. Yamashita, J. Polym. Sci., Part $A, 3,2165$ (1965).

8. K. Meyersen, R. C. Schulz, and W. Kern, Makromol. Chem., 58, 204 (1962).

9. F. A. Bovey and G. V. D. Tiers, Fortschr. Hochpolym.-Forsch., 3, 139 (1963).

10. A. E. Gillam and E. S. Stern, "An Introduction to Electronic Absorption Spectroscopy in Organic Chemistry" 2nd ed, Edward Arnord Publishers Ltd., London, 1957, p 70.

11. E. R. Blout and M. Fields, J. Amer. Chem. Soc., 70, 189 (1948).

12. T. Ouchi and M. Oiwa, Kogyo Kagaku Zasshi (J. Chem. Soc. Japan, Ind. Chem. Sect.), 72, 1587 (1969).

13. I. Takemura and H. Sumitomo, Abstracts, SPSJ 18th Symposium, Tokyo, Nov., 1969, p 39.

14. L. J. Young and C. C. Kennedy in "Copolymerization," G. E. Ham, Ed., John Wiley \& Sons, Inc., New York, N. Y., 1964, p 845. 